Kommentar

\section{Zitrone «Bologna»}

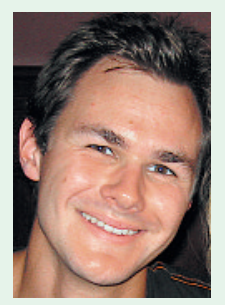

Bologna. Fast schon dämonisch schwebt dieser Begriff über unserem dritten Jahreskurs in Basel. Mal muss er als Erklärung dafür herhalten, dass wir unsere wöchentlich ändernden Stundenpläne immer erst auf den letzten Drücker erhalten, mal wischen sich die Kliniker den Schweiss von der Stirn, wenn sie realisieren, dass wir die Grundlagen ihrer klinischen Vorlesung noch nicht behandelt haben und seufzen: «Ach, ihr seid ja der Bologna-Jahreskurs fangen wir nochmal von vorne an.»

Zweifelsohne haben sich viele qualifizierte Personen mit der Einführung des Bologna-Systems an unserer medizinischen Fakultät befasst, doch scheint es, dass die meisten Ideen nicht ganz zu Ende gedacht wurden, oder wenn doch, für die Studierenden nicht verständlich sind. Was sind genau die Änderungen, welche den Wechsel zum Bologna-System so monumental machen? Irgendwie kann das niemand so genau sagen, abgesehen davon, dass Professoren meist ein Gesicht machen, als hätten sie in eine Zitrone gebissen, wenn das Wort fällt.

Während die Studierenden anderer Fakultäten seit Bologna auf einer heiteren ECTS*-Kreditpunktejagd sind, bei welcher oft eher «Aufwand pro ver- dienten Punkt» als Interesse am Thema die Einschreibungen beeinflusst, können die Mediziner nichts wählen. Kreditpunkte gibt es für die MCPrüfung, den praktischen OSCE-Test** und das Portfolio. Keine Chance, die viel gelobte Interdisziplinarität und Mobilität der schönen neuen Welt «Bologna» zu nutzen. Für fremde Inhalte gibt es keine Punkte, was sich leicht verstehen lässt, wenn man sich vorstellt, dass ein zukünftiger Arzt statt Chirurgie des Abdomens nach Belieben auch frühsteinzeitliche Töpferkunst besuchen könnte ... Somit bleibt für geschätzte $97 \%$ aller Medizinstudenten/-innen alles beim Alten: Büffeln, Kreditpunkte sammeln und am Ende das Staatsexamen bestehen. Ein Wechsel nach dem Bachelortitel an andere Universitäten gestaltet sich mindestens gleich schwierig wie früher, denn die Curricula sind weit entfernt von einer Angleichung. Summa summarum lässt sich aus Sicht eines Medizinstudenten sagen: Bologna bringt uns die Klinik in die Vorklinik (oft auf Kosten der Grundlagenlehre), eine ganze Menge Verwirrung und Kommentare von Ärzten wie «Also später werd' ich mich niemals von einem Arzt mit Staatsexamen 2012 behandeln lassen - ihr wart doch der erste Bologna-Kurs».

Marius Schlienger, Medizinstudent im dritten Jahreskurs an der Universität Basel

\title{
«Das Bologna-System soll die Sellbständigkeit fördern»
}

Frau Prof. Dr. med. Hedwig J. Kaiser ist seit 2002 Studiendekanin der Medizinischen Fakultät an der Universität Basel. Als Präsidentin der Bologna-Kommission der Schweizerischen Medizinischen Interfakultätskommission (SMIFK) hat sie die Einführung des Bologna-Systems in der Medizin an vorderster Front begleitet und mitgeprägt. Im folgenden Interview äussert sie sich im Sinne einer kurzen Bestandesaufnahme zu einigen zentralen Aspekten der Reform.

Interview:

Marionna Cathomas
Frau Kaiser, welches war der Hauptgrund für die Einführung des Bologna-Systems?

Der Entscheid zur Einführung des Bologna-Systems fiel auf politischer Ebene. Die Umsetzung selber wurde an die Rektorenkonferenz der Schweizerischen Universitäten (CRUS) delegiert. Die Schweizerische Medizinische Interfakultätskommission (SMIFK) gründete die Bologna-Kommission, welche von mir präsidiert wurde und das heutige Konzept des Bologna-Systems ausarbeitete. Ich möchte betonen, dass das BolognaSystem von allen medizinischen Fakultäten der Schweiz gemeinsam eingeführt wurde.

Welche Ziele stehen beim Bologna-System im Vordergrund? Die Hauptziele sind vergleichbare universitäre Abschlüsse, um die Mobilität zwischen den verschiede- 
nen Universitäten zu ermöglichen und zu fördern. Das Bologna-System beruht auf drei Säulen, dem Bachelor, Master und Doktorat. Schwerpunkte sind die stärkere Ausrichtung der Ausbildung auf die Berufsbefähigung sowie die Einführung des Kreditpunktsystems (ECTS European Credit Transfer System). Damit sollte neben der Vergleichbarkeit der studentischen Leistung auch die Mobilität garantiert werden. Für die Berufsbefähigung waren in der Medizin keine wesentlichen Änderungen notwendig, da die medizinische Ausbildung schon vor dem Bologna-System stark darua war. Jedoch führte die Überarbeitung des Curriculums zu einer verstärkten Sensibilisierung bezüglich Fragen der Berufsbefähigung.

Waren bei der Einführung des Bologna-Systems grössere Widerstände zu überwinden?

Bei der Ausarbeitung des Bologna-Systems waren alle fünf Schweizer Fakultäten sowie Vertreter der FMH, des Verbands Schweizerischer Assistenz- und Oberärztinnen und -ärzte (VSAO) und der Studierenden beteiligt. Somit war das Grundkonzept des Bologna-Systems von Anfang an breit abgestützt. Wie bei jeder Veränderung gab es natürlich auch kritische Stimmen.

Eine nächste Herausforderung, welche sich abzeichnet, ist die höhere Akzeptanz des Bachelor of Medicine. In Zukunft muss es möglich sein, mit dem Bachelor of Medicine berufstätig werden zu können, natürlich nicht als Arzt, sondern z. B. in der Pharmaindustrie. Dazu muss jedoch der Numerus Clausus angepasst werden, um genügend Ärzte zu haben, welche den Master of Medicine absolvieren.

Hatten Sie persönlich Bedenken bei der Einführung des Bologna-Systems?

Nein, ich hatte keine Bedenken. Als Präsidentin der SMIFK-Kommission konnte ich den Weg des BolognaSystems mitbestimmen und stand hinter den Beschlüssen.

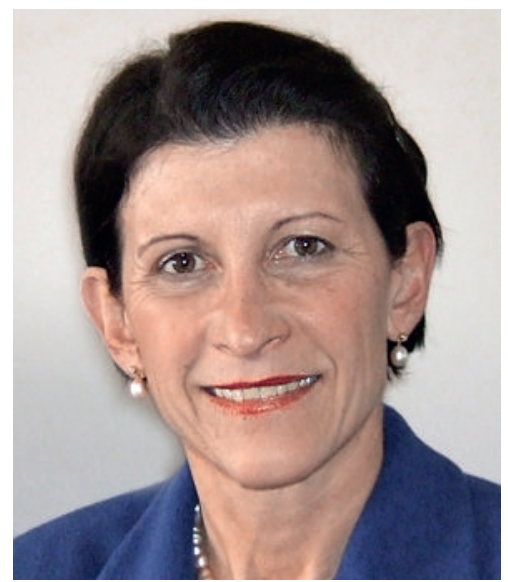

Keine Bedenken bei der Einführung des Bologna-Systems: Prof. Dr. med. Hedwig Kaiser, Präsidentin der BolognaKommission der Schweizerischen Medizinischen Interfakultätskommission (SMIFK)

\section{Inwiefern benachteiligt?}

Beispielsweise, weil sie die älteren Studenten nicht mehr um Rat fragen können, worauf sie im entsprechenden Themenblock achten müssen, und auch die kursierenden Prüfungsfragen nur noch eingeschränkte Gültigkeit haben. Zudem besteht schon die Tendenz, dass die Studierenden unselbstständiger werden. In den ersten 2 Jahren werden sie noch stark begleitet. Ab dem 3. Studienjahr sollten sie schon selbständiger sein, und wir servieren den Studierenden nicht mehr alles auf dem «silbernen Tablett». Das verunsichert sie, und sie beschuldigen das BolognaSystem. Genau in diesem Bereich liegt aber ein wesentliches Ziel: Das Bologna-System soll die Selbständigkeit fördern. Es gibt aber auch sehr positive Stimmen von Studierenden. Von Seiten der Dozenten habe ich übrigens nur wenig Negatives in Bezug auf die Einführung des neuen Systems gehört, obwohl das Bologna-System gerade für sie einen Mehraufwand

\section{«... wir servieren den Studierenden nicht mehr alles auf dem «silbernen}

\section{Tablett>. Das verunsichert sie, und sie beschuldigen das Bologna-System»}

Wie sehen Sie die Akzeptanz bei den Studentinnen und Studenten?

Ein Teil der Studenten hat eine Abneigung gegenüber dem Bologna-System, einzelne reagieren sogar ziemlich aggressiv. Diese Reaktionen haben aber weniger mit dem System zu tun, sondern vielmehr mit der Tatsache, dass einige Studierende das Gefühl haben, dass sie benachteiligt seien. bedeutet. Es gibt aber natürlich auch von Dozentenseite Kritik an den neuen studentenzentrierten Lehrformaten, aber das hat per se nichts mit der BolognaUmstellung zu tun, sondern wurde schon in der ersten Studienreform implementiert.

Würden Sie das Bologna-System wieder einführen? (Lacht) Ja, sicher. Das liegt in der Natur der Sache. 